\title{
Quantitative Assessment of Emphysema, Air Trapping, and Airway Thickening on Computed Tomography
}

\author{
Young Kyung Lee $\cdot$ Yeon-Mok Oh $\cdot$ Ji-Hyun Lee $\cdot$ Eun Kyung Kim • \\ Jin Hwa Lee · Namkug Kim · Joon Beom Seo · Sang Do Lee · \\ KOLD Study Group
}

Published online: 29 May 2008

(C) Springer Science+Business Media, LLC 2008

\section{Erratum to: Lung}

\section{DOI 10.1007/s00408-008-9071-0}

Below is the correct Figure 4B for the above-mentioned article, published online on March 20, 2008.

The online version of the original article can be found under doi:10.1007/s00408-008-9071-0.

Y. K. Lee · N. Kim · J. B. Seo $(\bowtie)$

Department of Radiology, Asan Medical Center, University of Ulsan College of Medicine, Research Institute of Radiology, 388-1, Pungnap-dong, Songpa-ku, Seoul 138-736, Korea e-mail: seojb@amc.seoul.kr; joonbeom.seo@gmail.com

\section{Y. K. Lee}

Department of Radiology, Bundang CHA Hospital, University of Pocheon Jungmoon College of Medicine, 183-8 Yatap-dong, Bundang-Ku, Seongnam-City, Gyeonggi-Do 463-712, Korea

\section{Y.-M. Oh · S. D. Lee ( $\square)$}

Division of Pulmonary and Critical Care Medicine, Department of Internal Medicine, Asan Medical Center, University of Ulsan College of Medicine, Research Institute of Radiology,

388-1, Pungnap-dong, Songpa-ku, Seoul 138-736, Korea

e-mail: sdlee@amc.seoul.kr

\section{J.-H. Lee · E. K. Kim}

Department of Internal Medicine, Bundang CHA Hospital,

University of Pocheon Jungmoon College of Medicine,

183-8 Yatap-dong, Bundang-Ku, Seongnam-City,

Gyeonggi-Do 463-712, Korea

J. H. Lee

Department of Internal Medicine, Ewha Womans University Mokdong Hospital, College of Medicine, Ewha Womans

University, Seoul, Korea

KOLD Study Group

Seoul, Korea

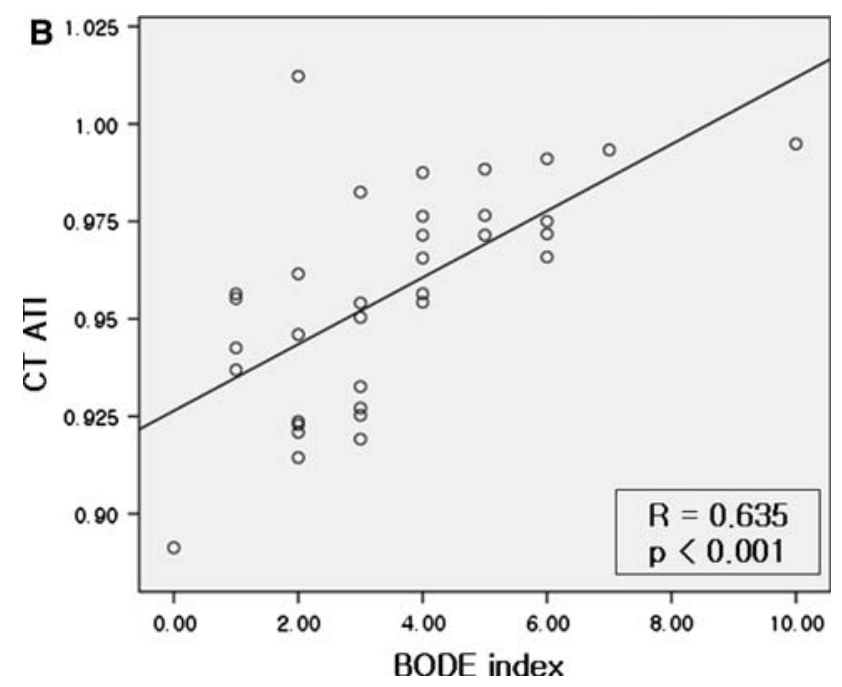

Fig. 4 